\title{
Primary User Signal Type Recognition Algorithm of Cognitive Radio Networks based on Active Learning in Building Indoors
}

\author{
Wang Xin* \\ School of Information \& Control Engineering \\ Shenyang Jianzhu University \\ Shenyang, China \\ e-mail: arnold0110@ sina.com \\ * Corresponding Author \\ Gao Zhijun \\ Graduate School \\ Shenyang Jianzhu University \\ Shenyang, China \\ Kan Fenglong \\ School of Information \& Control Engineering \\ Shenyang Jianzhu University \\ Shenyang, China \\ Guo Peng \\ Party School of Liaoning \\ Shenyang, China
}

\author{
Qi Yuanwei \\ School of Information \& Control Engineering \\ Shenyang Jianzhu University \\ Shenyang, China \\ Lü JiuYi \\ School of Information \& Control Engineering \\ Shenyang Jianzhu University \\ Shenyang, China \\ Li Hongwei \\ School of Information \& Control Engineering \\ Shenyang Jianzhu University \\ Shenyang, China \\ Wang Bin \\ Northeastern University at Qinhuangdao \\ Qinhuangdao, China
}

\begin{abstract}
Primary user signal modulation type recognition performance of building indoor environment has been the focus of attention and research in low signal-to-noise ratio. In this paper, a method based on active learning and support vector machine (SVM) for the primary user signal modulation type recognition is proposed in low signal to noise ratio. Three spectral coherence characteristic parameters are chosen via spectral correlation analysis, and the training samples and testing samples are formed for classification. Then, active learning algorithm is applied to obtain samples improved classification through a number of iterations, and SVM is formed. Finally, the formed SVM is utilized to recognize the primary user signal modulation type. Compared to the existing methods including the classifiers based on MME and ANN, the proposed approach is more effective in the case of low SNR and limited training numbers. The results show that the validity and superiority of the proposed algorithm on primary user signal modulation type recognition in building indoor environment.
\end{abstract}

Keywords-Active Learning; Primary User Signal Recognition; Support Vector Machine; Building Indoors; Spectral correlation analysis

\section{INTRODUCTION}

With the rapidly development of wireless communication technologies and the emergence of the new information technologies, a lot of important theoretical foundations and technical approaches has been applied to solve the problem in the buildings indoor environment. Among the information technologies, cognitive network has been recognized the key technology and method to utilize spectrum source effectively in the building indoor environment [1].

As one of the most important issues in cognitive radio networks[2,3], spectrum sensing is proposed to solve the shortage of vacant spectrum in recent. Many sensing techniques have been proposed over the last decade, based on energy detection, matched filter detection and cyclostationary feature detection algorithm[4,5,6]. The above algorithm for the primary user signal spectrum sensing have been achieved a certain results in low SNR conditions, but in the signal to noise ratio lower than $15 \mathrm{~dB}$ environment, the sense performance is not significant $[7,8]$. In view of the radio channel complex environment under the building indoor, spectrum sensing technology focused on the primary user signal spectrum sense problem under low signal-to-noise ratio circumstances of channel multi-path fading and shadow fading $[9,10]$.

Support vector machine, the representative of large mar-gin classifiers, enjoys a sound theoretical foundation based on Structural Risk Minimization [11,12]. It has achieved many successes in various empirical applications thanks to its superior generalization performance. Active learning, known as pool-based active learning, is an 
interactive learning technique designed to reduce the labour cost of labeling in which the learning algorithm can freely assign the unlabeled data instances to the training set. The basic idea is to select the most informative data in-stances for labeling by the users in the next learning round. In other words, the strategy of active learning is to select an optimal set of unlabeled data instances that minimizes the expected risk of the next round.

In this article, spectrum sensing is studied from the machine learning and an primary user signal recognition method based on active learning and support vector machine is proposed. A set of received signal spectrum features are extracted the important feature vector as training samples and testing samples for classification. Then, active learning algorithm is applied to obtain samples improved classification. Finally, we utilize the trained SVM to recognize the primary user signal modulation type in the building indoor environment.

The remainder of the paper is organized as follows. In section 2, the system model is described. The extraction of characteristic parameters is introduced in section 3. The proposed algorithm is investigated in section 4 and is well validated with computer simulation in section 5 . The several concluding remarks are made in section 6 .

\section{SYSTEM MODEL}

In this paper, we assume that a cognitive radio network with $\mathrm{W}$ primary users and $\mathrm{M}$ secondary users in building indoor environment, for any one of the secondary user, the presence of the primary user can be summarized as a hypothesis test model of two elements

$$
\left\{\begin{array}{c}
H_{0}: y(t)=n(t) \\
H_{1}: y(t)=\sum_{w=1}^{W} s_{w}(t)+n(t)
\end{array}\right.
$$

Where $H_{0}$ denotes that the absence of primary user, $H_{l}$ denotes that the presence of primary user. A process $s_{w}(t)$, that is any one of primary users signals, is said to be cyclostationary in building indoor environment if its mean and variance are periodic with a period $T ; n(t)$ represents the additive white Gauss noise, the mean is zero, variance is $\sigma_{n}^{2}$ 。

\section{PRimary USER Signal ReCOGNITION Algorithm BASED ON ACTIVE LEARNING}

In order to analyze the cyclostationary features of the received signal $y(t)$, two key functions are typically utilized. The cyclic autocorrelation function (CAF) is used for time domain analysis, which can be expressed as

$$
R^{\alpha}(\tau)=\frac{1}{T_{0}} \int_{0}^{T_{0}} R(t, \tau) \exp (-2 \pi \alpha t j) d t
$$

where $a$ is cyclic frequency, $\alpha=1 / T_{0}, T_{0}$ is cycle period, $\mathrm{R}(\mathrm{t}, \tau)$ is time-varying cyclic autocorrelation function of $y(t)$.

The spectral correlation function (SCF), which exhibits the spectral correlation of the signal $y(t)$, is obtained from the Fourier transform of the cyclic autocorrelation in Equation (2).

$$
S^{\alpha}(f)=\int_{-\infty}^{\infty} R^{\alpha}(\tau) \exp (-2 \pi \alpha t j) d t
$$

Based on the calculation of the spectral correlation function, we can obtain three parameters as characteristic parameters of a signal modulation identification ,which listed as follow:

Based on the calculation of the spectral correlation function, we can obtain three parameters as characteristic parameters of a signal modulation identification ,which listed as follow:

1. The largest value of all spectral correlation function

We assume that $\mathrm{S}(\mathrm{k})$ is the largest value of all spectral correlation function $S^{\alpha}(f)$ as the first extracted characteristic parameter of a received signal.

2. The correlation coefficient for the SCF

The correlation coefficient for the SCF between frequency components $f \pm 2 \alpha$, which is known as spectral coherence coefficient (SCC), can be calculated as

$$
C_{y}^{\alpha}(f)=\frac{S^{\alpha}(f)}{[S(f+\alpha / 2) S(f-\alpha / 2)]^{\frac{1}{2}}}
$$

3. The spectrum energy $\varepsilon$

When the received signal has more than one circular frequency,

the largest energy spectrum is the spectrum energy $\varepsilon$, which is defined as

$$
E=\frac{1}{K} \sum_{k=0}^{K-1}|S(k)|^{2}
$$

The above characteristic parameters are formed characteristic vector as the input samples.

In this section, a novel approach to recognize the primary user signal modulation type is introduced based on the proposed system model.

We have extracted the cyclic spectrum characteristic parameters of the presence of the primary user or not $\left(a_{1}^{q}, a_{2}^{q}, \cdots, a_{M}^{q}\right), \quad$ and obtain characteristic vector $y_{q}=\left(a_{1}^{q}, a_{2}^{q}, \cdots, a_{M}^{q}\right)^{T}, q=1,2, \cdots, M \quad$ as $\quad \mathrm{SVM}$ training samples, thus we generate SVM and using it to test input samples. Primary user signal recognition algorithm based on active learning can be expressed as follow. 


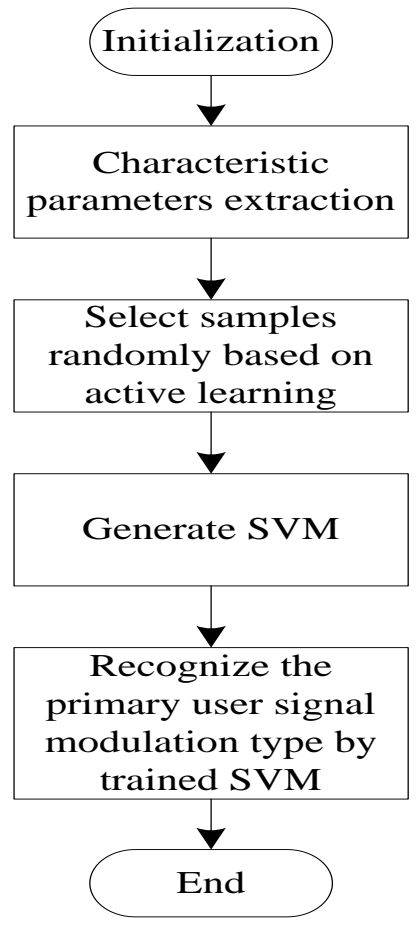

Figure 1. The flow chart of primary user signal recognition algorithm based on active learning

1. A few samples selected randomly from sampleset $U$ are marked manual tags and constitutes a training sample set L.

2. Training samples are randomly selected from the training set $\mathrm{L}$ to train the classifier, then establishing the initial classifer.

3.According to the standard Q query, A number of samples of the never marked samples in sample sets $U$ are selected and annotated, then placed in a training set $\mathrm{L}$.

4.Assume eigenvectors are collected as the positive training samples in the presence of the primary user, in which any of eigenvectors represents

$$
X_{1}^{i}=\left(E_{1}^{i}, M_{c 1}^{i}, M_{s 1}^{i}\right)^{T}, i=1,2, \cdots, Q_{1},
$$

and

$$
X_{0}^{i}=\left(E_{0}^{i}, M_{c 0}^{i}, M_{s 0}^{i}\right)^{T}, i=1,2, \cdots, Q_{0}
$$

as the negative training samples in the absence of the primary users.

5. A training set will be composed of the positive samples and negative samples, and utilizing the samples of training set to training SVM.

6. Repeat above steps, the primary user signal modulation type can be recognized by utilizing trained SVM.

The flow chart of primary user signal recognition algorithm based on active learning can be described as Figure 1.

\section{SimUlation AND ANALYSIS}

In order to verify that this algorithm in building indoor environment quality, using Matlab7.0 to complete experiments for 3 types of modulation signal (AM,BPSK, MSK). AM modulated signals are made by single frequency sine wave and BPSK, MSK are generated by a random sequence. To recognize the 3 modulation types, simulations are carried out with 1000 samples at SNR ranging from $-15 \mathrm{~dB}$ to $5 \mathrm{~dB}$, the length of DFT cyclic spectrum is 512 , where the parameter $C=50, \sigma=0.875$ are selected in cross-validation method of SVM.

Figure 2 shows the accuracy signal recognition rate of the different algorithms for AM, it is observed that the three algorithms have better signal recognition rate. The accuracy decreases with decreases of signal-to-noise ratio, the accuracy rate of MME algorithm is $19.2 \%, \mathrm{ANN}$ is

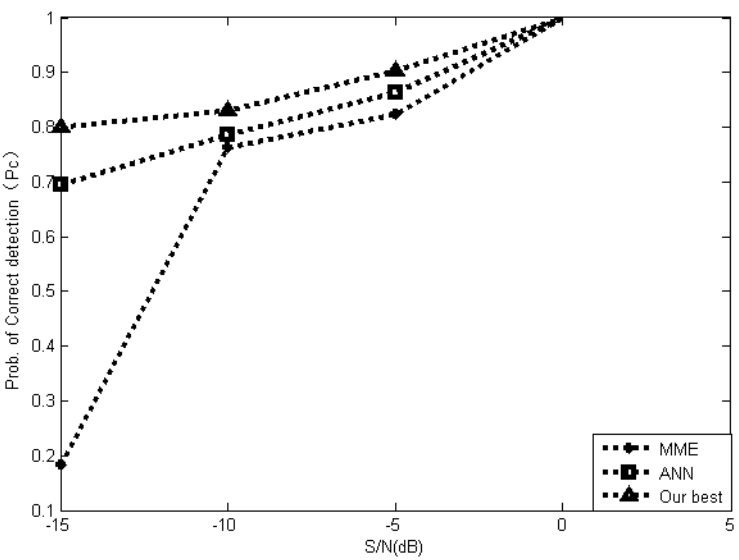

Figure 2. The accuracy rate of the different algorithms for AM

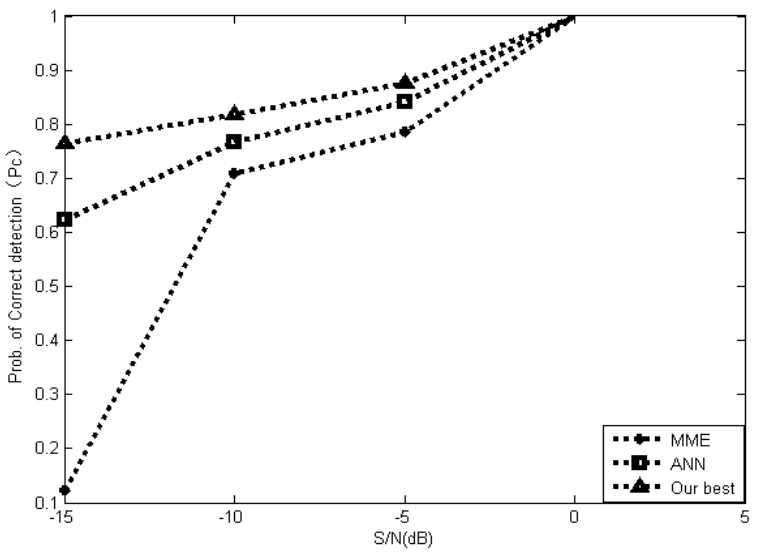

Figure 3. The accuracy rate of the different algorithms for BPSK

$70.1 \%$, but the proposed algorithm accuracy rate is still higher then $80.2 \%$ in $\mathrm{S} / \mathrm{N}=-15 \mathrm{~dB}$. Above results show that signal recognition performance of the proposed algorithm is significantly higher than the other two algorithms.

Figure 3 and Figure 4 show the accuracy rate of the different algorithms for BPSK and MSK respectively. Simulation results indicate that the MME algorithm detects the correct rate is very low, ANN algorithm for second when the signal-to-noise ratio is low. With the signal-to-noise ratio increased, the detection accuracy rate has improved, but the accuracy rate the above algorithms are still lower then our algorithm. 


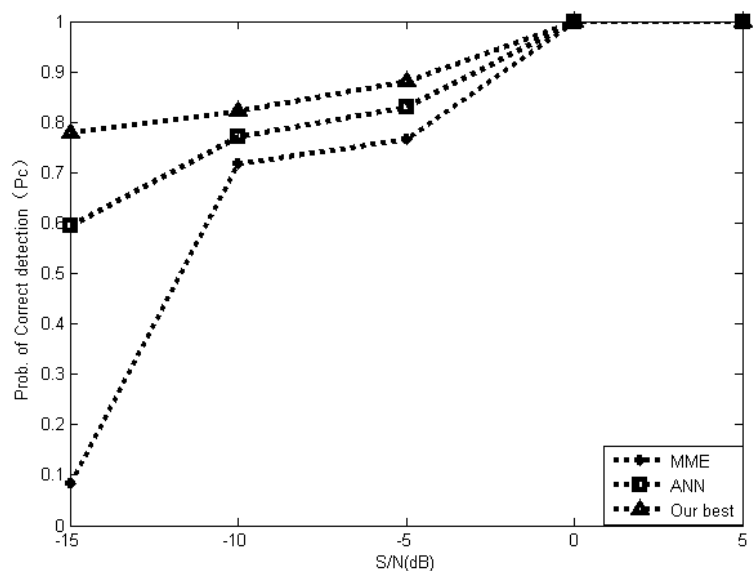

Figure 4. The accuracy rate of the different algorithms for MSK

The results show that the proposed algorithm has better primary user signal recognition performance in low signal-to-noise ratio environment, which demonstrates the feasibility of our algorithm.

\section{CONCLUSIONS}

This paper focuses on the primary user signal detection issue in low SNR environment, presented a primary signal detection algorithm based on AdaBoost.The algorithm utilizes spectral correlation analysis in signal feature extraction and AdaBoost algorithm to treat primary user signal detection. Simulation results show that this algorithm can better improve the spectrum sensing performance of the primary user signal in low signal-tonoise ratio environment.

\section{ACKNOWLEDGMENT}

This work was financially supported by the National Natural Science Foundation of China under Grant (No.61305125).

\section{REFERENCES}

[1] Wang X., Wang J.K., Liu Z.G. Spectrum sensing algorithm based on random forest in cognitive network. Chinese Journal of Scientific Instrument. 2013, Vol. 34, no. 11, pp. 35-41.

[2] Devroye, N., Vu, M., and Tarokh, V., "Cognitive radio networks". IEEE Signal Processing Mag., Nov. 2008, vol. 25, pp. 12-23.

[3] Yucek, T., and Arslan, H., "A survey of spectrum sensing algorithms for cognitive radio applications" .IEEE Commun. Surveys Tutorials, quarter. 2009, vol. 11, no.1, pp. 116-130.

[4] Wang, B., and Liu, K. J. R. "Advances in cognitive radio networks: A survey”. IEEE J. Sel. Topics Signal Process, Feb. 2011,vol. 5, no. 1 , pp. 5-23.

[5] Haykin, S., Thomson, D., and Reed, J. "Spectrum sensing for cognitive radio". Proc. IEEE, May 2009, vol. 97, no. 5, pp. 849877.

[6] Ghasemi, A., and Sousa, E., "Spectrum sensing in cognitive radio networks: requirements, challenges and design trade-offs". IEEE Commun. Mag, Apr. 2008, vol. 46, no. 4, pp. 32-39.

[7] BHARGAVI, D., and MURTHY, C. R. "Performance comparison of energy, matched-filter and cyclostationarity based spectrum sensing".2010 IEEE Eleventh International Workshop on Signal Processing Advances in Wireless Communications (SPAWC). 2010. pp.1-5.

[8] ZENG Y H, LIANG Y C. "Eigenvalue-based spectrum sensing algorithms for cognitive radio". IEEE Transactions on Communications, 2009, vol. 57, no. 6, pp.1784-1793.

[9] He, A., Bae, K. K., and Newman, T., "A survey of artificial intelligence for cognitive radios". IEEE Trans. Veh. Technol., May 2010, vol. 59, no. 4, pp. 1578-1592.

[10] Dong, X., Li, Y., and Wu, C. "A learner based on neural network for cognitive radio". in 12th IEEE International Conference on Communication Technology (ICCT '10), Nanjing, China, Nov 2010, pp. 893-896.

[11] Cortes,C. and Vapnik, V. "Support-vector networks" . Machine Learning, 1995, 20, pp.273-297.

[12] Hu, H., Song.J., and Wang, Y. "Signal classification based on spectral correlation analysis and SVM in cognitive radio". in Advanced Information Networking and Applications, 2008. AINA 2008. 22th International Conference on, Mar 2008, pp. 883-887. 